Relations industrielles

Industrial Relations

\title{
Work in America et le Québec
}

\section{Charles Gagné}

Volume 29, numéro 1, 1974

URI : https://id.erudit.org/iderudit/028487ar

DOI : https://doi.org/10.7202/028487ar

Aller au sommaire du numéro

Éditeur(s)

Département des relations industrielles de l'Université Laval

ISSN

0034-379X (imprimé)

1703-8138 (numérique)

Découvrir la revue

Citer cet article

Gagné, C. (1974). Work in America et le Québec. Relations industrielles /

Industrial Relations, 29(1), 218-221. https://doi.org/10.7202/028487ar

Tous droits réservés (C Département des relations industrielles de l'Université Laval, 1974
Ce document est protégé par la loi sur le droit d'auteur. L’utilisation des services d'Érudit (y compris la reproduction) est assujettie à sa politique d'utilisation que vous pouvez consulter en ligne.

https://apropos.erudit.org/fr/usagers/politique-dutilisation/ 


\section{WORK IN AMERICA ET LE QUÉBEC}

\section{Charles Gagné *}

\section{LE RAPPORT «WORK IN AMERICA 》}

Il est révélateur qu'au moment où on est assailli de littérature, soit sur l'augmentation des heures de loisir, soit sur la nécessité de décloisonner l'école de l'expérience du vécu ${ }^{1}$, on se retrouve subitement en face d'un ouvrage qui rappelle que le travail demeure encore l'élément fondamental de la vie, non seulement parce qu'il en occupe le plus clair du temps, aussi et surtout, parce qu'il est porteur des éléments qui permettent à l'individu de se réaliser comme ... de se détériorer.

A première vue, on pourrait penser qu'il s'agit d'un autre essai paru dans une quelconque revue savante, mais ce n'est pas le cas puisqu'il s'agit du Rapport ${ }^{2}$ d'un groupe de travail où on retrouve les noms de James O'Toole, Neal Herrick, Elliot Liebow, Harold Sheppard, préparé à l'intention d'Elliot $\mathrm{L}$. Richardson, secrétaire à la santé, à l'éducation et au bien-être du gouvernement des U.S.A., et qui comporte une série d'applications bien concrètes.

Le Rapport s'inscrit dans le contexte de l'amélioration des conditions de travail. Ce n'est pas un document qui rebrasse à neuf les concepts déjà usés de participation par objectif ou d'enrichissement des tâches. Il dépasse et escalade en proposant ce qu'il appelle une redéfinition des tâches (Redesign of Jobs) et la participation aux bénéfices. On dira, peut-être, que la participation aux bénéfices n'est pas non plus une idée neuve. La nouveauté vient plutôt du fait qu'on commence à l'appliquer chez nos voisins qui comprennent, de plus en plus, qu'il s'agit d'un facteur d'intéressement et de hausse de productivité. Les auteurs de l'ouvrage présentent ces mesures comme étant une arme très efficace pour lutter contre le chômage dans un contexte inflationniste.

L'ouvrage contient sept chapitres, dont le premier traite de la notion de travail, de son caractère central dans la vie, de la difficulté et de l'importance d'en prendre conscience, des recherches faites sur le sujet, etc., etc. . . Le travail, la santé, le bien-être, la stabilité de la famille, l'éducation sont intimement reliés dans la vie. Tout tend à démontrer que le

* Gagné, C., directeur, service des politiques, Direction générale de la maind'œuvre, ministère du Travail et de la Main-d'œuvre du Québec. Cet article ne représente pas la position officielle du ministère du Travail et de la Main-d'œuvre du Québec.

1 Edgar Faure, Apprendre à être, Paris, Unesco-Fayard (1972).

2 Work In America, Report of a special task force, Washington, HEW, December 1972, 178 pages. 
travail a un impact réel et dominant sur ces secteurs. Agir sur le travail c'est agir sur le reste.

Il existe à l'heure actuelle un écart entre les conditions de travail et l'attitude des travailleurs : les conditions ont évolué moins rapidement que les attitudes. Résultat : sur cent cols blancs, quarante-trois choisiraient le même métier si c'était à refaire, et le pourcentage descend à vingt-quatre dans le cas des cols bleus. La conséquence : dans l'état actuel des choses, l'impact du travail sur le reste se traduit par un bilan négatif.

Les deux chapitres suivants portent sur la conception que différentes catégories de main-d'œuvre se font du travail aux U.S.A. et sur la relation et les coûts qui existent entre le travail et la santé, (travail-longévité, travail-maladie du cœur, travail-santé mentale). Les catégories inventoriées sont les cols bleus, cols blancs, les gérants, les jeunes travailleurs, les immigrants, la femme au travail, les travailleurs âgés et retraités.

Le Rapport suggère que l'efficacité de toute action de type "redesign of jobs » impliquant les conditions de travail est conditionnelle à une prise de conscience par les autorités politiques, patronales et syndicales de l'impact de la situation de travail sur les coûts de la santé et de la nécessité d'y prendre des mesures préventives.

Les chapitres IV et V traitent d'une formule toute nouvelle applicable sur les lieux de travail et du rôle respectif de l'entreprise, des syndicats et de l'Etat. Il est aussi question de l'impact de la formation sur le travail. La formule s'appelle la redéfinition des tâches (Redesign of Works). Elle est appliquée dans plusieurs entreprises, avec comme résultat beaucoup plus de satisfaction au travail, en même temps qu'une croissance de la productivité de 5 à $40 \%$. On y donne aussi différents modes d'interventions de l'Etat, tout en soulignant qu'il n'en coûterait pas plus cher au contribuable.

$\mathrm{Du}$ côté de la formation, on propose quelques programmes bien précis et on rejoint le «Rapport Faure 》 sur bien des points quand on suggère que le but de l'éducation devrait être de maintenir la curiosité, promouvoir la confiance en soi, stimuler la soif d'apprendre et développer la compétence, lesquelles sont tous des besoins ressentis par les travailleurs. «L'éducation, est-il dit, devrait faire elle-même l'objet d'une expérience de redéfinition des tâches $\gg$.

Dans les chapitres suivants, il est question des différentes stratégies de l'Etat en matière de plein emploi, de la formation de la main-d'œuvre et les politiques de bien-être.

\section{L'APPLICABILITÉ DU RAPPORT AU QUÉBEC}

L'Etat a été «forcé 》 de mettre en chantier des programmes de toutes sortes basés sur l'initiative et les entreprises ont reçu béatement des subventions. 
La main-d'œuvre au Québec, dans son ensemble, a beaucoup plus de points en commun avec la main-d'œuvre américaine qu'avec celle du reste du monde, tant du point de vue du management, de l'organisation que des méthodes de production etc... C'est pourquoi, il y a de fortes chances que les déboires de nos voisins du sud aient ici aussi de fortes résonnances.

Le Québec est au prise depuis une bonne décade avec toute une série de problèmes reliés au travail. Celui qui a le plus retenu l'attention a été la concomitance d'un taux élevé de chômage en même temps qu'une certaine pénurie de main-d'œuvre, ce qui a fait dire à certains que cet état était dû à la paresse des travailleurs.

La réplique de l'Etat a été de mettre en œuvre toute une série de programmes destinés : soit à la sécurité du revenu, prestations d'assistance chômage, d'assistance sociale, soit à la création d'emplois, dégrèvements d'impôts, subventions à la création d'emplois, aide à l'investissement, programmes d'initiatives locales, perspective jeunesse ou soit des programmes de formation professionnelle.

Face à la persistance du phénomène, malgré ces mesures, l'Etat a vu fondre ses réserves d'assistance chômage et a dû utiliser des méthodes coercitives de retour au travail, tandis que de l'autre côté plusieurs compagnies se voyaient accusées de Corporate Welfare Bums. Au même moment, l'Etat faisait un succès de ses programmes d'initiatives de toutes sortes.

L'expérience qui vient de se jouer ici indique que le travail ne répugne pas à la main-d'œuvre en chômage, puisqu'elle a participé massivement aux projets d'initiatives. De là à trouver la cause dans des conditions de travail qui ont intéressé les travailleurs, il n'y a qu'un pas. Ceci nous amène aussi à nous demander si la baisse de la clientèle des cours de formation professionnelle ne vient pas du fait qu'on n'est pas intéressé à retourner au travail pour y retrouver des conditions qui n'ont pas changé. Bien plus, l'Etat, en créant des programmes attrayants, a attiré davantage de main-d'œuvre du circuit régulier du travail, plutôt que d'employer, en exclusivité, les véritables chômeurs, et on sait ce qui a suivi ... des enquêtes etc.

On pourrait tout résumer en disant que l'Etat vient de faire la preuve qu'en agissant sur les conditions de travail il est possible d'intéresser et de faire travailler bien du monde et que, par conséquent, ce n'est pas le travail qui répugne.

Work in America aura au moins le mérite de nous indiquer que la meilleure mesure préventive contre le chômage c'est d'agir sur les conditions de travail, de façon qu'elles stimulent l'intérêt du travailleur.

Dans les années à venir, l'Etat ferait bien d'y voir à deux fois au lieu de donner béatement des subventions à l'entreprise et de prendre 
sur soi les «drop-out » du circuit du travail. Le problème, il est au coeur de l'entreprise, il se nomme «conditions de travail », et c'est là que doit porter le plus clair des interventions de l'Etat.

\section{LE DILEMME BIEN RÉEL DES ENTREPRISES}

Les entreprises du Québec, à l'image de ce qui se passe ailleurs, doivent acquitter d'année en année une note croissante de coûts sociaux. Le ministre des Affaires sociales du Québec disait dans le Devoir du 3 avril dernier qu'au rythme de croissance du coût des services de santé au Québec, c'est tout le produit national brut des années 2000 qui sera consacré à ce seul poste.

Face à ceci, l'entreprise n'a pas le choix ; ou bien elle se réfugie dans un statu quo stérile et accepte passivement de payer une facture annuelle d'impôt de plus en plus élevée au risque, comme le rappelle le ministre des Affaires sociales, d'y consacrer toutes les ressources de l'entreprise, ce qui est ridicule. Ou bien elle accepte de prendre sa part des responsabilités sociales, s'engage résolument dans une réflexion, telle que le propose Work in America, et se décide à faire, dès aujourd'hui, ce qu'on pourrait qualifier d'investissements sociaux préventifs en agissant sur les conditions de travail.

La question qui se pose porte sur la volonté de l'entreprise de s'engager d'elle-même dans cette nouvelle voie. Le passé récent nous enseigne qu'il est bien plus facile de se faire accorder des subventions d'investissement jusqu'à l'équivalent des impôts à payer qu'autrement (Corporate Welfare Bums).

Il serait grandement temps que l'Etat fasse prendre conscience à l'entreprise de l'importance d'agir sur les conditions de travail. Ceci pourrait se faire sous forme de subventions.

\section{POLITIQUES DE MAIN-D'CEUVRE : ÉVALUATION DE L'EXPÉRIENCE QUÉBÉCOISE}

Introduction (JEAN-PAul Deschênes). Les politiques de main-d'oeuvre et le développement socio-économique (Pierre Harvey). Les programmes de maind'oeuvre et leur évolution (ÉMILIEN LANDRY et LouIs LEMIEUX). La convention collective et les politiques de main-d'oeuvre ( $Y$ VES Dubé et Jean-Paul DEschÊNES). Les politiques de main-d'oeuvre et la formation professionnelle des adultes (Pierre Paquet). Le maintien du revenu (Gilles Beausolem). L'évaluation des programmes de main-d'oeuvre: résultats et pertinence. La coordination des politiques de main-d'oeuvre (ClaUde Mérineau).

$$
1 \text { volume, } 188 \text { pages - Prix : } \$ 5.50
$$

\section{LES PRESSES DE L'UNIVERSITÉ LAVAL}

G1K 7 R4 Québec, P.Q. Canada 